\title{
Growth differentiation factor-15 as candidate predictor for mortality in adults with pulmonary hypertension
}

\author{
Laurie W Geenen, ${ }^{1}$ Vivan J M Baggen, ${ }^{1}$ Robert M Kauling, ${ }^{1}$ Thomas Koudstaal, ${ }^{2}$ \\ Karin A Boomars, ${ }^{2}$ Eric Boersma, ${ }^{\oplus 1,3}$ Jolien W Roos-Hesselink, ${ }^{\oplus}$ \\ Annemien $\mathrm{E}$ van den Bosch ${ }^{\oplus}$
}

\begin{abstract}
- Additional material is published online only. To view please visit the journal online (http://dx.doi.org/10.1136/ heartjnl-2019-315111).

${ }^{1}$ Department of Cardiology, Erasmus University Medical Centre, Rotterdam, The Netherlands

${ }^{2}$ Department of Pulmonary Diseases, Erasmus University Medical Centre, Rotterdam, The Netherlands

${ }^{3}$ Department of Clinical Epidemiology, Erasmus University Medical Centre, Rotterdam, The Netherlands
\end{abstract}

\section{Correspondence to}

Dr Annemien E van den Bosch, Department of Cardiology,

Erasmus University Medical

Centre, Rotterdam 3000 CA, The Netherlands:

a.e.vandenbosch@erasmusmc.n

Received 24 March 2019 Revised 12 August 2019 Accepted 19 August 2019

Check for updates

(C) Author(s) (or their employer(s)) 2019. No commercial re-use. See rights and permissions. Published by BMJ.

To cite: Geenen LW Baggen VJM, Kauling RM, et al. Heart Epub ahead of print: [please include Day Month Year]. doi:10.1136/ heartjnl-2019-315111

\section{ABSTRACT}

Objective Despite its predictive value for mortality in various diseases, the relevance of growth differentiation factor-15 (GDF-15) as prognostic biomarker in pulmonary hypertension $(\mathrm{PH})$ remains unclear. This study investigated the association between GDF-15 and outcomes in adults with $\mathrm{PH}$.

Methods This is a single-centre prospective observational cohort study. All adults with PH were included at the day of their diagnostic right heart catheterisation between 2012 and 2016. PH due to left heart disease was excluded. Venous blood sampling was performed and included GDF-15 and N-terminal proB-type natriuretic peptide (NT-proBNP) measurements. Kaplan-Meier curves and Cox regression analysis were used to investigate the association between GDF-15 and a composite endpoint of death or lung transplantation. We adjusted for age and NT-proBNP in multivariable analysis. Reference values were established by GDF-15 measurements in healthy controls.

Results GDF-15 was measured in 103 patients (median age 59.2 years, 65\% women, 51\% pulmonary arterial hypertension). GDF-15 was elevated in 76 patients (74\%). After a median follow-up of 3.4 (IQR 2.3-4.6) years, 32 patients (31.1\%) reached the primary endpoint. Event-free survival 2 years after diagnosis was $100 \%$ in patients with normal GDF-15 versus $72.4 \%$ in patients with elevated GDF-15 ( $p=0.007)$. A significant association was found between GDF-15 and the primary endpoint (HR per twofold higher value 1.77, $95 \% \mathrm{Cl} 1.39$ to $2.27, \mathrm{p}<0.001$ ), also after adjustment for age and NT-proBNP (HR 1.41, 95\% Cl 1.02 to 1.94, $\mathrm{p}=0.038$ )

Conclusions High GDF-15 levels are associated with an increased risk of death or transplant in adults with $\mathrm{PH}$, independent of age and NT-proBNP. As non-specific biomarker, GDF-15 could particularly be useful to detect low-risk patients.

\section{INTRODUCTION}

Growth differentiation factor-15 (GDF-15) is a member of the transforming growth factor-B cytokine superfamily and is known for its role in cell growth and differentiation. ${ }^{1}$ Animal models have shown that GDF-15 is induced in response to cardiac pressure overload, ischaemia, oxidative stress and reperfusion injury. ${ }^{23}$ However, GDF-15 is non-tissue specific; secretion of GDF-15 is produced by a wide variety of cells ${ }^{4}$ and is seen in high concentrations in various diseases. ${ }^{5}$ It may therefore not be surprising that GDF-15 has been identified as predictor for all-cause mortality in a wide spectrum of diseases: cardiovascular diseases, ${ }^{67}$ various types of cancers ${ }^{89}$ and pulmonary diseases. ${ }^{10} 11$ To that end, the use of GDF-15 in risk stratification has been doubted because of its lack of disease specificity. ${ }^{12}$ Paradoxically, non-tissue specific biomarkers could offer a solution in some diseases in which heterogeneity and concomitant disease have a considerable influence on the prognosis.

Pulmonary hypertension $(\mathrm{PH})$ is a disease in which heterogeneity introduces major challenges in risk stratification. $\mathrm{PH}$ is defined by an increased pulmonary artery pressure of $\geq 25 \mathrm{~mm} \mathrm{Hg}^{13}$ which eventually leads to right ventricular failure and mortality. However, underlying aetiology of increased pulmonary pressures includes a wide spectrum of diseases. The prognosis of $\mathrm{PH}$ strongly depends on the aetiology. ${ }^{14} \mathrm{~A}$ recent study including $\mathrm{PH}$ of all aetiologies showed that only in $23.8 \%$ of the patients right ventricular failure was the main cause of death and other causes such as respiratory failure, malignancy, sepsis and infection were not uncommon. ${ }^{15}$ In this perspective, GDF-15 could be an ideal prognostic biomarker for mortality risk stratification in $\mathrm{PH}$, as it may reflect not only cardiac failure but also incorporate more disease processes.

Therefore, this study aimed to investigate levels of GDF-15 in adults with PH of different aetiologies at the time of diagnosis and to determine its association with prognosis. A cohort of healthy volunteers was used to establish reference values for GDF-15.

\section{METHODS}

\section{Patient and public involvement}

Patients or the public were not involved in the design, or conduct, or reporting, or dissemination of our research.

\section{Study design}

This prospective observational cohort study aimed to include all consecutive adults diagnosed with $\mathrm{PH}$ in our tertiary centre between May 2012 and October 2016. The diagnostic work-up of PH consisted of an inpatient visit during which the following tests were performed: physical examination by a cardiologist 


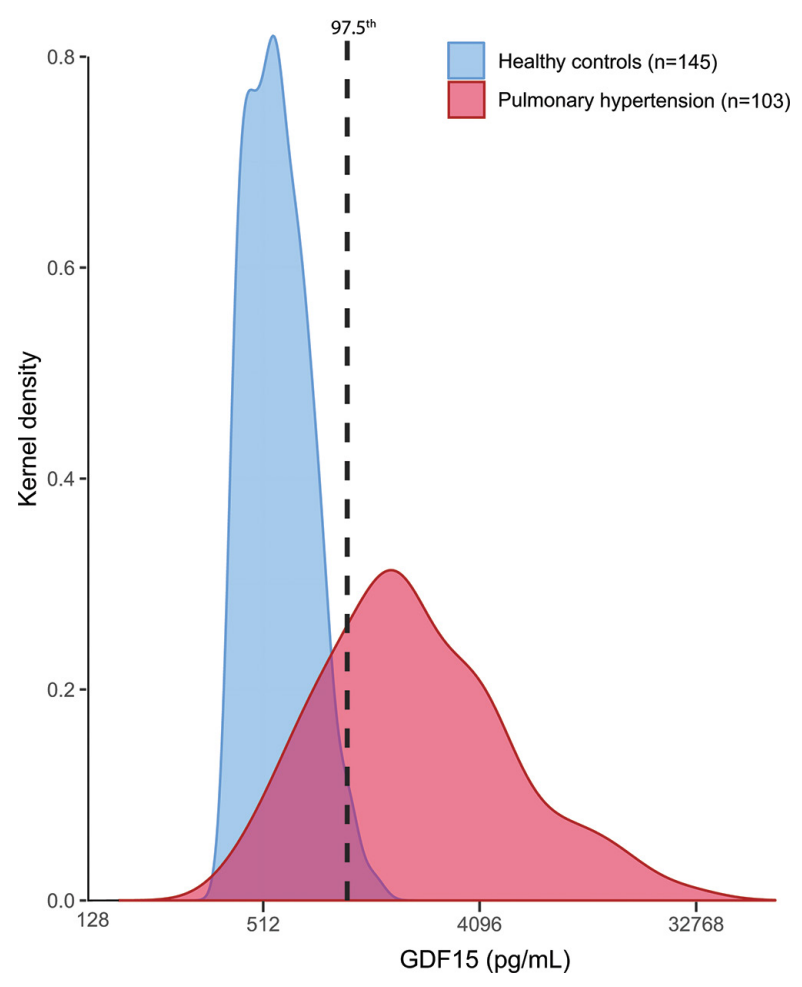

Figure 1 Kernel density plot showing the distribution of growth differentiation factor-15 (GDF-15) in healthy controls and in adults with pulmonary hypertension. The 97.5th percentile of the GDF-15 distribution in the healthy controls is indicated by the black-dotted line. The $\mathrm{x}$-axis is shown on the 2-log scale.

and pulmonary physician, 6 min walking test, 12-lead ECG, transthoracic echocardiography, venous blood sampling, cardiac CT and right heart catheterisation. Baseline was defined as the day of the diagnostic right heart catheterisation. Diagnosis of $\mathrm{PH}$ was defined as a mean pulmonary artery pressure $\geq 25 \mathrm{~mm}$ $\mathrm{Hg}$. Exclusion criteria were unconfirmed diagnosis of $\mathrm{PH}$ due to an incomplete diagnostic work-up, not $\mathrm{PH}$ treatment-naive, $<18$ years, not capable of understanding or signing informed consent and $\mathrm{PH}$ due to left heart disease. The study protocol was approved by the local medical ethics committee and is conform to the principles outlined in the Declaration of Helsinki.

Classification of PH was done in accordance with the WHO classification: pulmonary arterial hypertension (PAH), $\mathrm{PH}$ due to lung disease, chronic thromboembolic pulmonary hypertension (CTEPH) and $\mathrm{PH}$ with unclear/multifactorial mechanisms (WHO 5). PAH was further subdivided according to the classification. ${ }^{13} 16$ Self-declared healthy volunteers were recruited between January 2014 and December 2014 to serve as control cohort. All volunteers underwent physical examination, ECG, echocardiography and venous blood sampling on the same day. More detailed information has been previously published. ${ }^{17}$

\section{Data collection}

Transthoracic echocardiography was performed using a commercially available ultrasound system (ie, 33, Philips Medical Systems, Best, the Netherlands). The echocardiographic imaging analysis was performed in accordance with echocardiographic guidelines on cardiac chamber quantification. ${ }^{18}$ We used a SwanGanz catheter to obtain haemodynamic measurements during the right heart catheterisation. Fick's principle or thermodilution was used to measure cardiac output. On indication, a fluid challenge was performed to distinguish $\mathrm{PH}$ due to left heart disease from precapillary PH. Data were collected and stored in an online electronic case report form (PAHTool, version 4.3.5947.29411; Inovoltus, Santa Maria da Feira, Portugal). More details have been described previously. ${ }^{19}$

\section{Biomarker assessment}

Venous blood sampling was performed during the diagnostic right heart catheterisation and was for study purposes only. N-terminal pro-B-type natriuretic peptide (NT-proBNP) was directly measured from fresh blood samples with the use of an electrochemiluminescence assay (Roche Diagnostics) in the clinical chemistry laboratory. The rest of the serum samples were aliquoted and stored in our biobank by $-80^{\circ} \mathrm{C}$. GDF-15 was determined in thawed serum samples by batch analysis with electrochemiluminescence immunoassay using the Cobas 6000 analyser (Roche Diagnostics, Basel, Switzerland). Limit of detection was $400 \mathrm{pg} / \mathrm{mL}$. GDF-15 was measured once in all patients with $\mathrm{PH}$. In healthy controls, GDF-15 was measured twice to assess reproducibility and to establish reference values.

\section{Definition of endpoints}

Endpoints were defined prior to data collection. The primary endpoint was defined as all-cause mortality or lung transplantation. The secondary endpoint was a composite endpoint including the elements of the primary endpoint and heart failure. Heart failure was defined as any hospitalisation due to heart failure with requirement for uptitration or initiation of diuretics.

Protocolled half-yearly visits to the outpatient clinic were scheduled to guarantee data on endpoints. Patients were treated in accordance with the European Society of Cardiology (ESC) guidelines. ${ }^{13} \mathrm{PH}$ medication was prescribed when indicated and patients with CTEPH eligible for pulmonary endarterectomy or balloon pulmonary angioplasty were referred to a specialised centre.

Patient records and the municipal personal records database were used to adjudicate the endpoints until 1 January 2019 without the knowledge of any biomarker level.

\section{Statistical analysis}

Continuous variables are presented as mean \pm SD or median (IQR). Differences in baseline characteristics between patients with a normal and elevated GDF-15 were investigated with the Student's t-test or Mann-Whitney test $(n<30)$ for continuous variables. Differences in categorical variables were investigated with the $\chi^{2}$ test or Fisher exact test. Correlations between GDF-15 and clinical characteristics were assessed with Pearson or Spearman correlation.

Reproducibility of GDF-15 assay was visualised by a Bland-Altman plot and the coefficient of variation and intraclass correlation coefficient were calculated. The 97.5 th percentile based on 2-log transformed GDF-15 distribution in healthy controls was used to define an elevated GDF-15 level. GDF-15 distributions were visualised using a Kernel density plot.

GDF-15 and NT-proBNP levels were 2-log transformed because of skewed distributions. Missing data were taken care of using multiple imputation with five imputations considering all meaningful variables as predictor, including the endpoints. Endpoints were not imputed. Pooled estimates were obtained based on Rubin's rule. ${ }^{20}$ Survival curves were derived using the Kaplan-Meier estimator and compared with the log-rank test. Cox-proportional hazard regression was used to assess associations with endpoints in univariable and multivariable analysis. 
Table 1 Baseline characteristics of all patients and subdivided to patients with a normal GDF-15 and elevated GDF-15 at baseline

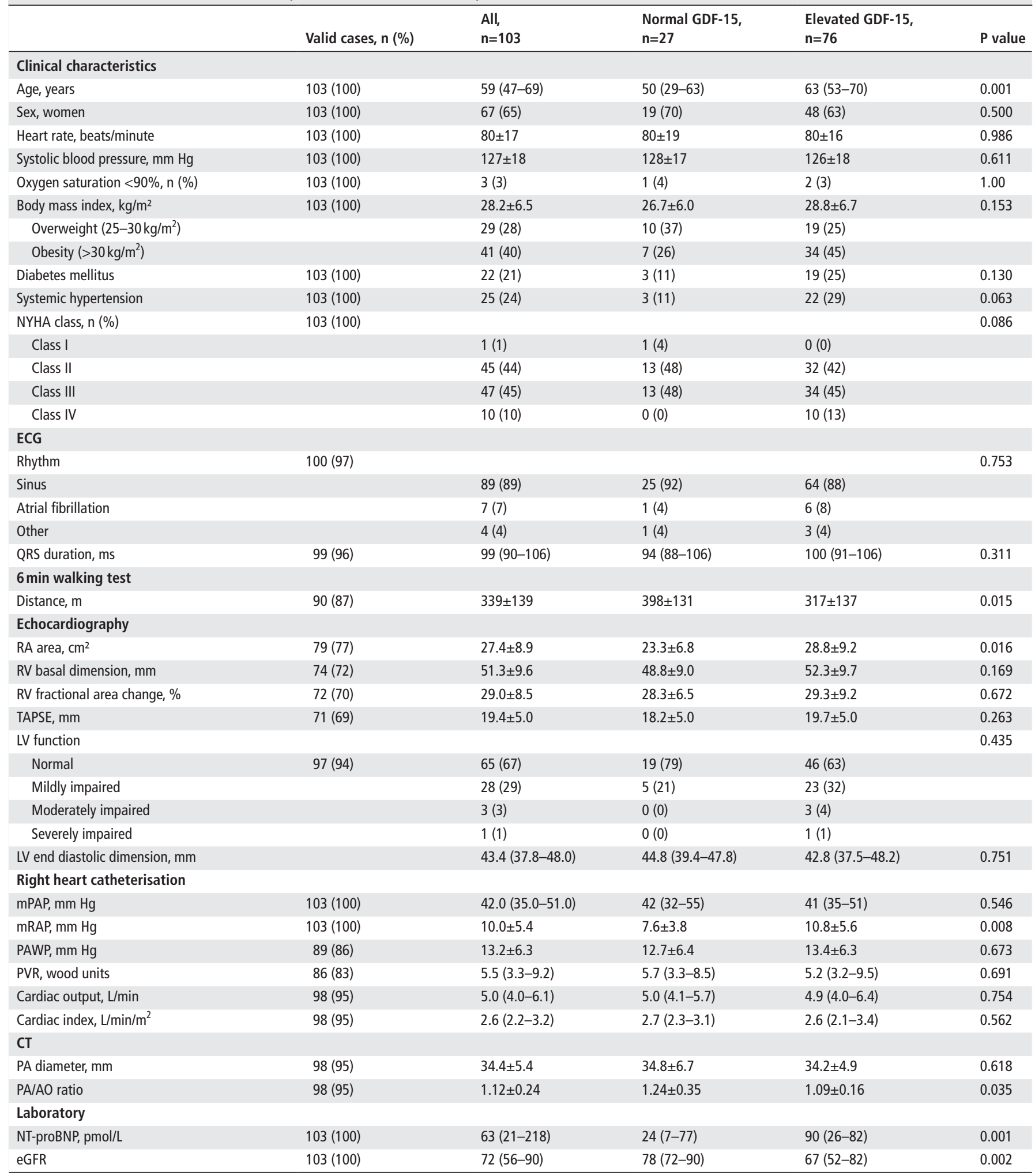

Elevated GDF-15 was defined as: GDF-15 $>920 \mathrm{pg} / \mathrm{mL}$ (age $<50$ years) or GDF- $15>1330 \mathrm{pg} / \mathrm{mL}$ (age $>50$ years).

AO, aorta; eGFR, estimated glomerular filtration rate; GDF-15, growth differentiation factor-15; LV, left ventricular; mPAP, mean pulmonary artery pressure;mRAP, mean right atrial pressure; NT-proBNP, N-terminal pro B-type natriuretic peptide;NYHA, New York Heart Association; PA, pulmonary artery; PAWP, pulmonary artery wedge pressure;PVR, pulmonary vascular resistance; RA, right atrial; RV, right ventricular; TAPSE, tricuspid annular plane systolic excursion.

The proportional hazard assumption was assessed through Schoenfield residual plots and through proportional hazard tests. We adjusted all analyses for age because we considered this as an important confounder. Due to the limited number of events, we adjusted for only one additional clinical variable at a time in additional analyses. A subgroup analysis was performed restricted to patients with $\mathrm{PAH}$, with adjustment for only one clinical variable at a time because of a limited number of events. 


\begin{tabular}{|c|c|c|}
\hline & \multicolumn{2}{|l|}{ GDF-15 } \\
\hline & $r$ & $P$ value \\
\hline \multicolumn{3}{|l|}{ Clinical characteristics } \\
\hline Age & 0.39 & $<0.001$ \\
\hline Sex & 0.03 & 0.802 \\
\hline Body mass index & 0.02 & 0.859 \\
\hline Heart rate & 0.15 & 0.128 \\
\hline Systolic blood pressure & -0.21 & 0.032 \\
\hline Oxygen saturation <90\% & -0.09 & 0.380 \\
\hline NYHA class & 0.28 & 0.004 \\
\hline Diabetes & 0.31 & 0.001 \\
\hline Systemic hypertension & 0.30 & 0.002 \\
\hline \multicolumn{3}{|l|}{ ECG } \\
\hline Loss of sinus rhythm & 0.10 & 0.316 \\
\hline QRS duration & 0.09 & 0.362 \\
\hline \multicolumn{3}{|l|}{6 min walking test } \\
\hline Distance & -0.44 & $<0.001$ \\
\hline \multicolumn{3}{|l|}{ Echocardiography } \\
\hline Right atrial area & 0.16 & 0.174 \\
\hline RV basal dimension & 0.08 & 0.487 \\
\hline RV fractional area change & -0.05 & 0.651 \\
\hline TAPSE, mm & -0.04 & 0.743 \\
\hline LV function & 0.15 & 0.143 \\
\hline LV end diastolic dimension & -0.15 & 0.187 \\
\hline \multicolumn{3}{|l|}{ Right heart catheterisation } \\
\hline mPAP & -0.01 & 0.899 \\
\hline mRAP & 0.21 & 0.032 \\
\hline PAWP & -0.06 & 0.564 \\
\hline Pulmonary vascular resistance & 0.18 & 0.091 \\
\hline Cardiac output & -0.11 & 0.272 \\
\hline Cardiac index & -0.18 & 0.083 \\
\hline \multicolumn{3}{|l|}{$\mathrm{CT}$} \\
\hline PA diameter & -0.081 & 0.427 \\
\hline $\mathrm{PA} / \mathrm{AO}$ ratio & -0.30 & 0.002 \\
\hline \multicolumn{3}{|l|}{ Laboratory } \\
\hline NT-proBNP & 0.51 & $<0.001$ \\
\hline eGFR & -0.47 & $<0.001$ \\
\hline
\end{tabular}

Significant correlations are in bold. 2-log transformed GDF-15 levels were used for the analysis.

AO, aorta; eGFR, estimated glomerular filtration rate; GDF-15, growth differentiation factor-15; LV, left ventricular; mPAP, mean pulmonary artery pressure; mRAP, mean right atrial pressure; NT-proBNP, N-terminal pro B-type natriuretic peptide; NYHA, New York Heart Association; PA, pulmonary artery; PAWP, pulmonary artery wedge pressure; RV, right ventricular; TAPSE, tricuspid annular plane systolic excursion.

To evaluate the potential predictive value of GDF-15 beyond age and NT-proBNP, we determined C-indices of models with and without GDF-15. The likelihood ratio test was used to compare models.

Statistical analyses were performed using IBM SPSS Statistics (version 24) and $\mathrm{R}$ version 5.5.3. A two-sided p-value $<0.05$ was considered statistically significant.

\section{RESULTS}

\section{Reproducibility of GDF-15 assay and reference values}

GDF-15 was measured twice in 145 healthy controls. Reproducibility of the assay was good, with coefficient of variation of $0.68 \%$, limits of agreement of -15.49 to $23.60 \mathrm{pg} / \mathrm{mL}$ and intraclass correlation coefficient of $0.99(0.99-1.00, \mathrm{p}<0.001)$ (online supplementary file 1). Levels ranged from $400 \mathrm{pg} / \mathrm{mL}$
GDF-15 tertile distribution

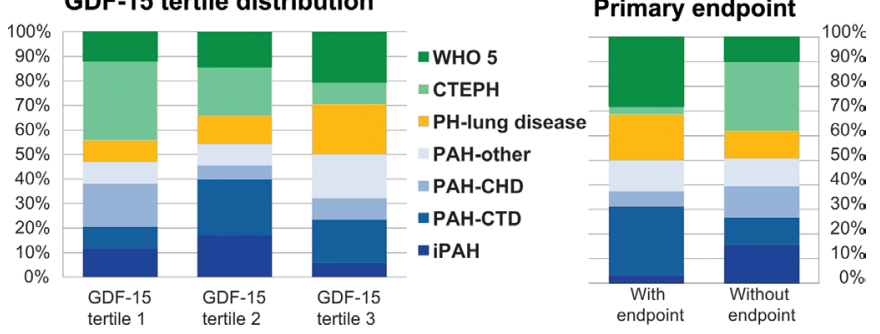

Figure 2 Distribution of $\mathrm{PH}$ subdiagnoses according to a normal or elevated GDF-15 and according to the primary endpoint. Elevated GDF-15 was defined as: $>920 \mathrm{pg} / \mathrm{mL}$ (age $<50$ years) or $>1330 \mathrm{pg} /$ $\mathrm{mL}$ (age $>50$ years). Diagnosis groups are in accordance with the ESC guidelines of PH. PAH-other consisted of: pulmonary veno-occlusive disease $(n=2)$, PAH-associated with portal hypertension $(n=7)$, hereditary PAH $(n=2)$, drug-induced and toxin-induced PAH $(n=1)$. CTEPH, chronic thromboembolic pulmonary hypertension; GDF-15, growth differentiation factor-15; iPAH, idiopathic pulmonary arterial hypertension; PAH-CHD, pulmonary arterial hypertension due to congenital heart disease, $\mathrm{PAH}-\mathrm{CTD}$, pulmonary arterial hypertension due to connective tissue disease; $\mathrm{PH}$, pulmonary hypertension; $\mathrm{WHO}=\mathrm{WHO}$ group 5.

(lower limit of detection; $\mathrm{n}=15$ ) to $1495 \mathrm{pg} / \mathrm{mL}$. GDF-15 was not significantly different between men and women; however, there was a significant correlation with age $(\mathrm{r}=0.48, \mathrm{p}<0.001)$ (online supplementary file 2). Consequently, age-specific reference values were established: $>920 \mathrm{pg} / \mathrm{mL}$ for patients aged $<50$ years and $1330 \mathrm{pg} / \mathrm{mL}$ for patients aged $>50$ years. Distribution of GDF-15 levels in healthy controls and patients with $\mathrm{PH}$ is shown in figure 1.

\section{Baseline characteristics}

GDF-15 was measured in 103 out of 106 patients (97\%) who were originally included in this study (online supplementary file 2 ). In three cases, no serum sample was traceable to measure GDF-15. Median age was 59 (IQR 47-69) years, 67 were women $(65 \%)$ and 52 had PAH (51\%). Over half of the patients was in New York Heart Association (NYHA) functional class III (46\%) or IV (10\%). GDF-15 was above the limit of detection in all patients, with a median GDF-15 of 1974 (IQR 1096-4173)pg/ $\mathrm{mL}$. An elevated GDF-15 was found in 76 patients $(74 \%)$. Patients with normal GDF-15 were significantly younger, had a longer $6 \mathrm{~min}$ walking distance, a lower right atrial area and mean right atrial pressure, a better renal function and a lower NT-proBNP, than patients with elevated GDF-15 (table 1).

Higher GDF-15 significantly correlated with higher NYHA class, shorter $6 \mathrm{~min}$ walking distance and the presence of hypertension and diabetes mellitus. GDF-15 did not show any significant correlation with echocardiographic or invasive haemodynamic measures, except for the mean right atrial pressure. GDF-15 did show a significant moderate correlation with NT-proBNP $(r=0.51, p<0.001)$ and estimated glomerular filtration rate $(\mathrm{eGFR})(\mathrm{r}=-0.47, \mathrm{p}<0.001)$ (table 2).

There was no statistical difference in GDF-15 distributions across PH subgroups $(p=0.061)$ (online supplementary file 3 ). The distribution of different $\mathrm{PH}$ aetiologies across patients with normal versus elevated GDF-15 is presented in figure 2.

\section{Follow-up}

Follow-up data were $100 \%$ complete. Median follow-up was 3.4 (IQR 2.3-4.6) years. The primary endpoint was reached in 32 


\section{Primary endpoint}
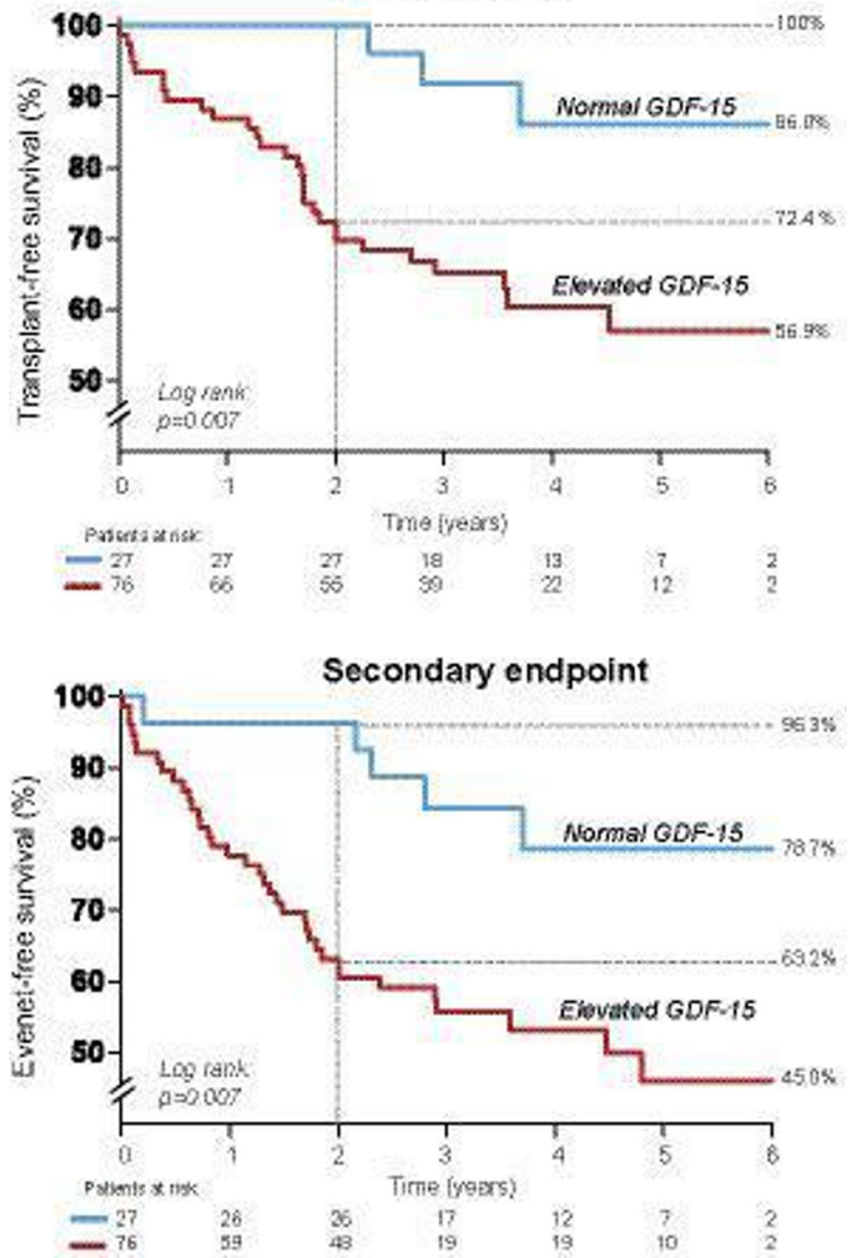

Figure 3 Survival curve demonstrating the cumulative transplant-free survival in patients with pulmonary hypertension with a normal growth differentiation factor-15 (GDF-15) level and patients with an elevated GDF-15 level. An elevated GDF-15 was defined as: GDF-15>920 pg/mL (age $<50$ years) or GDF-15 $>1330 \mathrm{pg} / \mathrm{mL}$ (age $>50$ years).

patients (31.1\%), and the secondary endpoint was reached in 41 patients $(39.8 \%)$. Figure 1 shows the distribution of $\mathrm{PH}$ aetiologies according to primary endpoint achievement. Considering all endpoints separately, 30 patients died, 4 patients underwent lung transplantation and 26 patients were hospitalised for heart failure. Causes of death were end-stage heart failure $(n=9)$, sudden death presumed cardiac $(n=4)$, multiorgan failure $(n=3)$, and other diverse causes $(n=13)$ described in detail in online supplementary file 5 . In one patient, cause of death was unknown. PAH-specific medication was initiated during follow-up in $90 \%$ of the patients with PAH $(n=47)$ and in $71 \%$ of the patients with CTEPH $(n=15)$. Five patients $(24 \%)$ with CTEPH underwent balloon pulmonary angioplasty and three patients (14\%) underwent surgical pulmonary endarterectomy.

\section{GDF-15 as prognostic biomarker}

Patients with normal GDF-15 had a significant higher transplant-free survival than patients with elevated GDF-15 $(p=0.007)$. This difference was specifically pronounced within the first 2 years of follow-up; patients with normal GDF-15 were all alive and free of transplantation up to 2 years after diagnosis, compared with $72.4 \%$ of the patients with elevated GDF-15. Comparable results were found regarding the secondary endpoint (figure 3).

Analysed continuously, GDF-15 was significantly associated with the primary endpoint, also after adjustment for several clinical characteristics. After adjustment for age and NT-proBNP, GDF-15 was still significantly associated with the primary endpoint. Addition of GDF-15 to a model with NT-proBNP and age, significantly increased the C-index from 0.73 (95\% CI 0.65 to 0.81$)$ to $0.76(95 \%$ CI 0.69 to 0.83$)(\mathrm{p}=0.042)$.

GDF-15 was significantly associated with the secondary endpoint, although less strong compared with the primary endpoint. This association remained present after adjustment for clinical characteristics, but not after adjustment for age and NT-proBNP (table 3).

Subgroup analysis restricted to PAH showed an independent prognostic value of GDF-15 for the primary endpoint when adjusted for clinical variables, including NT-proBNP. Notably, adjustment for eGFR led to a non-significant result in this subgroup (online supplementary file 6).

\section{DISCUSSION}

In $76 \%$ of the patients with PH, GDF-15 is elevated at the time of the diagnosis. Higher GDF-15 levels were associated with an older age, higher NYHA class, shorter 6 min walking distance, higher mean right atrial pressure and higher NT-proBNP; however, no association was found with echocardiographic measurements.

Table 3 Association between GDF-15 and the primary and secondary endpoint

\begin{tabular}{|c|c|c|c|c|}
\hline & \multicolumn{2}{|c|}{ Primary endpoint $(n=32)$} & \multicolumn{2}{|c|}{ Secondary endpoint $(n=41)$} \\
\hline & $\mathrm{HR}^{*}(95 \% \mathrm{Cl})$ & $P$ value & $\mathrm{HR}^{*}(95 \% \mathrm{Cl})$ & $P$ value \\
\hline GDF-15 (univariable) & 1.77 (1.39 to 2.27$)$ & $<0.001$ & 1.58 (1.27 to 1.96$)$ & $<0.001$ \\
\hline \multicolumn{5}{|l|}{ Adjusted for: } \\
\hline Age and sex & 1.74 (1.34 to 2.26$)$ & $<0.001$ & 1.53 (1.21 to 1.93$)$ & $<0.001$ \\
\hline Age and NYHA class $3 / 4$ & 1.60 (1.23 to 2.08$)$ & $<0.001$ & 1.40 (1.11 to 1.76$)$ & 0.005 \\
\hline Age and 6-MWD & 1.57 (1.21 to 2.04$)$ & 0.001 & 1.31 (1.03 to 1.66$)$ & 0.025 \\
\hline Age and right atrial area & $1.71(1.32$ to 2.20$)$ & $<0.001$ & 1.49 (1.19 to 1.87$)$ & 0.001 \\
\hline Age and cardiac index & 1.77 (1.35 to 2.31$)$ & $<0.001$ & 1.53 (1.21 to 1.93$)$ & $<0.001$ \\
\hline Age and mRAP & 1.78 (1.37 to 2.32$)$ & $<0.001$ & 1.46 (1.15 to 1.86$)$ & 0.002 \\
\hline Age and eGFR & $1.72(1.28$ to 2.30$)$ & $<0.001$ & 1.48 (1.14 to 1.92$)$ & 0.003 \\
\hline Age and NT-proBNP & 1.41 (1.02 to 1.94$)$ & 0.038 & $1.21(0.91$ to 1.62$)$ & 0.192 \\
\hline
\end{tabular}

*HR per twofold higher value of GDF-15.

eGFR, estimated glomerular filtration rate;GDF-15, growth differentiation factor-15; mRAP, mean right atrial pressure; 6-MWD, 6 min walking distance; NT-proBNP, N-terminal pro B-type natriuretic peptide; NYHA, NewYork Heart Association. 
GDF-15 was significantly associated with mortality or lung transplantation independent of age and NT-proBNP levels, and yielded an incremental predictive value. Moreover, a normal GDF-15 ruled out the risk of death or transplantation in the first 2 years after the diagnosis of $\mathrm{PH}$.

\section{Previous reports}

Data on GDF-15 in adults with $\mathrm{PH}$ are currently limited and are restricted to some specific PAH diagnoses. Nickel et al measured GDF-15 in 76 treatment-naive adults with idiopathic PAH (iPAH), 55\% of these patients had a GDF-15 level above $1200 \mathrm{ng} / \mathrm{L}$. Higher GDF-15 levels were associated with an increased risk of death or transplantation, independent of NT-proBNP and other clinical variables. ${ }^{10}$ Rhodes et al later confirmed the predictive value of GDF-15 for mortality in 139 patients with iPAH. ${ }^{21}$ Zelniker et al investigated GDF-15 in 96 non-treatment naive patients with PAH. The majority of these patients had $\mathrm{PAH}(68 \%)$ or PAH due to connective tissue disease (22\%). They found that higher GDF-15 levels were associated with the 4-year mortality risk. However, GDF-15 was not a better prognosticator than NT-proBNP, high-sensitive troponin-T and proatrial natriuretic peptide in their study. ${ }^{22}$

Cross-sectional studies have shown that GDF-15 is higher in patients with $\mathrm{PAH}$ due to systemic sclerosis compared with patients with systemic sclerosis free of $\mathrm{PAH} .{ }^{23}$ Moreover, higher levels of GDF-15 were found in patients with CTEPH compared with patients with a history of acute pulmonary embolism without development of CTEPH. ${ }^{24}$

To the best of our knowledge, this is the first study investigating GDF-15 in a group of mixed $\mathrm{PH}$ aetiologies. It showed that GDF-15 levels are considerably higher in adults with $\mathrm{PH}$ compared with a reference cohort and that a GDF-15 level within the reference range is associated with a low risk of mortality, transplantation or heart failure in adults with PH. GDF-15 could therefore be a promising biomarker. However, the price of the GDF-15-assay kit is still high and ideally biomarkers should be easily measured against a low price.

\section{Underlying mechanisms of GDF-15}

There are several possible explanations for the high GDF-15 levels in patients with $\mathrm{PH}$ and its strong association with mortality found in our study. First, since GDF-15 is involved in the regulation of cell processes and $\mathrm{PH}$ is characterised by pulmonary vascular endothelial remodelling, ${ }^{25}$ GDF-15 could reflect the process of vascular remodelling in $\mathrm{PH}$. This is supported by a study that found higher GDF-15 levels in the vascular endothelial cells of PH. ${ }^{26}$ Second, GDF-15 may be induced by myocardial cell stress caused by the increased right ventricular afterload as it has been shown that GDF-15 is induced in the myocardium of mice after exposure to ischaemic injury. ${ }^{2}$ Moreover, it has been suggested that GDF-15 is part of a cardioprotective pathway. ${ }^{3}$ Third, GDF-15 may have been secreted due to any concomitant disease in these patients such as systemic sclerosis or chronic obstructive pulmonary disease. Finally, it has been shown that GDF-15 levels are higher in patients with diabetes and other unfavourable cardiovascular risk factors. ${ }^{27}$ In our study, 21\% of the patients had diabetes mellitus and 24\% had systemic hypertension and both comorbidities correlated with higher GDF-15 levels.

Considering all of the above-mentioned reasons, it is most presumable that GDF-15 levels were influenced by more disease processes than solely cardiac involvement. This can be further supported by the fact that levels of GDF-15 did show an association with NYHA class and the 6 min walking distance, while associations with haemodynamic or echocardiographic measurements were mostly absent. Despite limited associations with cardiac pressure and function, GDF-15 did show a moderate positive correlation with NT-proBNP.

The prognosis of adults with $\mathrm{PH}$ is still very challenging, mainly due to heterogeneity and accompanied diseases in this population. Paradoxically, as we hypothesised, in this specific setting the low disease specificity of GDF-15 turns out advantageous and could serve as prognostic biomarker to detect patients with low-risk PH. Restricting the survival analysis to only patients with $\mathrm{PAH}$ showed approximately the same prognostic value. This strengthens the idea that GDF-15 could be a prognostic biomarker in patients with $\mathrm{PH}$ independent of the specific underlying aetiology. Furthermore, GDF-15 showed an independent association with the primary endpoint when adjusted for age and NT-proBNP, and provided an incremental predictive value.

\section{Limitations}

Blood sampling was performed during the diagnostic right heart catheterisation in treatment-naive patients with $\mathrm{PH}$. In patients with PAH and CTEPH, treatment was initiated directly after confirmation of diagnosis. We were not able to adjust for treatment effect in the association between GDF-15 and the endpoints. This study therefore reflects the association between GDF-15 and adverse outcomes for patients with $\mathrm{PH}$ treated in accordance to the ESC guidelines.

Patients with PH due to left heart disease were not included in this study, and this should be kept in mind when extrapolating the results to other studies. Also our study consisted of a heterogeneous group of $\mathrm{PH}$ aetiologies and subgroup analysis could only be performed for PAH. It should be kept in mind that clinical usefulness of GDF-15 might differ among PH aetiologies. Furthermore, this study focused only one a single prognosticator and due to the limited statistical power, the additive value of GDF-15 to existing risk scores could not be assessed. Conclusion with regard to the use of this biomarker in the context of current risk models, specifically concerning patients with $\mathrm{PAH}$, are therefore limited.

\section{Clinical implications}

GDF-15 showed a strong association with mortality in our study and specifically identified low-risk patients at the time of diagnosis of PH. Therefore, measuring GDF-15 may be considered in patients with newly diagnosed $\mathrm{PH}$ to identify, and subsequently reassure, low-risk patients. In these patients, the follow-up frequency could probably be lowered compared with high-risk patients. Since GDF-15 is not tissue-specific and concentrations can rise or decline in response to any disease process, GDF-15 seems not the most optimal biomarker to specifically pursue biomarker-guided therapy. Nevertheless, more data are needed to validate these findings before finding its way to daily clinical practice and larger studies with more power are needed to investigate GDF-15 in the light of current existing risk prediction models for PAH.

\section{CONCLUSIONS}

In adults with PH, a normal GDF-15 level at the time of diagnosis identifies patients with a very low 2-year risk of mortality or transplantation. GDF-15 could therefore be a promising biomarker to identify low-risk patients with normal GDF-15 levels. Due to its lack of tissue specificity, the role of GDF-15 
in future biomarker-guided therapy is uncertain and may be limited; however, GDF-15 could potentially serve as predictor for mortality in patients with $\mathrm{PH}$ of various aetiologies. Future studies, preferably including a larger cohort of patients with $\mathrm{PH}$, are recommended to further investigate this promising biomarker.

\section{Key messages}

What is already known on this subject?

- Growth differentiation factor-15 (GDF-15) is a non-tissue specific biomarker and has been identified as strong predictor for mortality in various disease. Risk stratification in pulmonary hypertension remains challenging because of the considerable heterogeneity in this disease population.

\section{What might this study add?}

- GDF-15 is significantly associated with the transplant-free survival in adults with pulmonary hypertension patients, independent of $\mathrm{N}$-terminal pro-B-type natriuretic peptide levels. A normal GDF-15 level at the time of diagnosis identifies patients with a very low 2-year mortality risk.

\section{How might this impact on clinical practice?}

- In adults with pulmonary hypertension, a GDF-15 measurement at the time of diagnosis should be considered to identify low-risk patients whom can be reassured.

Contributors All the authors contributed to one or more of the following: conception of design of the work (VJMB, KAB, EB, JWR-H, AEvdB), data collection (LWG, VJMB, RMK, TK, KAB, JWR-H, AEvdB), data analysis and interpretation ( $L W G, V J M B, E B$, JWR-H and AEvdB), drafting the manuscript (LWG, JWR-H and $A E v d B$ ) and critical revision of the manuscript for important intellectual content (all authors).

Funding Roche provided GDF-15 assays free of charge for the support of this investigator-initiated study.

Competing interests None declared.

Patient consent for publication Not required.

Provenance and peer review Not commissioned; externally peer reviewed.

Data availability statement Data are available on reasonable request.

\section{REFERENCES}

1 Paralkar VM, Vail AL, Grasser WA, et al. Cloning and characterization of a novel member of the transforming growth factor-beta/bone morphogenetic protein family. $J$ Biol Chem 1998;273:13760-7.

2 Kempf T, Eden M, Strelau J, et al. The transforming growth factor-beta superfamily member growth-differentiation factor- 15 protects the heart from ischemia/reperfusion injury. Circ Res 2006:98:351-60.

3 Xu J, Kimball TR, Lorenz JN, et al. GDF15/MIC-1 functions as a protective and antihypertrophic factor released from the myocardium in association with SMAD protein activation. Circ Res 2006;98:342-50.

4 Unsicker K, Spittau B, Krieglstein K. The multiple facets of the TGF- $\beta$ family cytokine growth/differentiation factor-15/macrophage inhibitory cytokine-1. Cytokine Growth Factor Rev 2013;24:373-84.
5 Mueller T, Leitner I, Egger M, et al. Association of the biomarkers soluble ST2, galectin-3 and growth-differentiation factor- 15 with heart failure and other noncardiac diseases. Clin Chim Acta 2015;445:155-60.

6 Sharma A, Stevens SR, Lucas J, et al. Utility of growth differentiation factor-15, a marker of oxidative stress and inflammation, in chronic heart failure: insights from the HF-ACTION Study. JACC Heart Fail 2017:5:724-34.

7 Lindholm D, James SK, Gabrysch K, et al. Association of multiple biomarkers with risk of all-cause and cause-specific mortality after acute coronary syndromes: a secondary analysis of the Plato biomarker study. JAMA Cardiol 2018;3.

8 Mehta RS, Chong DQ, Song M, et al. Association between plasma levels of macrophage inhibitory cytokine-1 before diagnosis of colorectal cancer and mortality. Gastroenterology 2015;149:614-22.

9 Staff AC, Trovik J, Eriksson AGZ, et al. Elevated plasma growth differentiation factor-15 correlates with lymph node metastases and poor survival in endometrial cancer. Clin Cancer Res 2011;17:4825-33.

10 Nickel N, Kempf T, Tapken H, et al. Growth differentiation factor-15 in idiopathic pulmonary arterial hypertension. Am J Respir Crit Care Med 2008;178:534-41.

11 Duran L, Kayhan S, Guzel A, et al. The prognostic values of GDF-15 in comparison with NT-proBNP in patients with normotensive acute pulmonary embolism. Clin Lab 2014;60:1365-71.

12 Lindahl B. The story of growth differentiation factor 15: another piece of the puzzle. Clin Chem 2013;59:1550-2

13 Galiè N, Humbert M, Vachiery J-L, et al. 2015 ESC/ERS guidelines for the diagnosis and treatment of pulmonary hypertension: the joint Task force for the diagnosis and treatment of pulmonary hypertension of the European Society of Cardiology (ESC) and the European Respiratory Society (ERS): endorsed by: Association for European Paediatric and Congenital Cardiology (AEPC), International Society for Heart and Lung Transplantation (ISHLT). Eur Heart J 2016:37:67-119.

14 Escribano-Subias P, Blanco I, López-Meseguer M, et al. Survival in pulmonary hypertension in Spain: insights from the Spanish registry. Eur Respir J 2012;40:596-603.

15 Gall H, Felix JF, Schneck FK, et al. The Giessen pulmonary hypertension registry: survival in pulmonary hypertension subgroups. J Heart Lung Transplant 2017;36:957-67.

16 Simonneau G, Gatzoulis MA, Adatia I, et al. Updated clinical classification of pulmonary hypertension. J Am Coll Cardiol 2013;62(25 Suppl):D34-41.

17 Menting ME, McGhie JS, Koopman LP, et al. Normal myocardial strain values using 2D speckle tracking echocardiography in healthy adults aged 20 to 72 years. Echocardiography 2016;33:1665-75.

18 Lang RM, Badano LP, Mor-Avi V, et al. Recommendations for cardiac chamber quantification by echocardiography in adults: an update from the American Society of Echocardiography and the European Association of Cardiovascular Imaging. Eur Heart J Cardiovasc Imaging 2015;16:233-71.

19 Geenen LW, Baggen VJM, Koudstaal T, et al. The prognostic value of various biomarkers in adults with pulmonary hypertension; a multi-biomarker approach. Am Heart J 2019:208:91-9.

20 Rubin DB. Inference and missing data. Biometrika 1976;63:581-92.

21 Rhodes CJ, Wharton J, Howard LS, et al. Red cell distribution width outperforms other potential circulating biomarkers in predicting survival in idiopathic pulmonary arterial hypertension. Heart 2011:97:1054-60.

22 Zelniker T, Uhlmann L, Spaich S, et al. Novel biomarkers for risk stratification in pulmonary arterial hypertension. ERJ Open Res 2015:1.

23 Meadows CA, Risbano MG, Zhang L, et al. Increased expression of growth differentiation factor-15 in systemic sclerosis-associated pulmonary arterial hypertension. Chest 2011;139:994-1002

24 Klok FA, Surie S, Kempf T, et al. A simple non-invasive diagnostic algorithm for ruling out chronic thromboembolic pulmonary hypertension in patients after acute pulmonary embolism. Thromb Res 2011;128:21-6.

25 Tuder RM, Cool CD, Yeager $M$, et al. The pathobiology of pulmonary hypertension. Endothelium. Clin Chest Med 2001;22:405-18

26 Nickel N, Jonigk D, Kempf T, et al. GDF-15 is abundantly expressed in plexiform lesions in patients with pulmonary arterial hypertension and affects proliferation and apoptosis of pulmonary endothelial cells. Respir Res 2011;12:62.

27 Ho JE, Mahajan A, Chen M-H, et al. Clinical and genetic correlates of growth differentiation factor 15 in the community. Clin Chem 2012:58:1582-91. 